\author{
Nataša Janićijević \\ Università di Belgrado - Facoltà di Filologia \\ natasa.janicijevic@fil.bg.ac.rs
}

\title{
VERBI DI POSTURA IN ITALIANO E IN SERBO ${ }^{1}$
}

\begin{abstract}
Il presente contributo si propone di analizzare da un punto di vista contrastivo i tre principali verbi e le relative espressioni verbali indicanti la posizione del corpo in italiano e in serbo: sedere, stare seduto vs sedeti, stare in piedi vs stajati e giacere, stare sdraiato/disteso vs ležati. Nel contributo vengono esaminate le proprietà semantiche di questi verbi nelle due lingue, con particolare riguardo al loro uso in funzione di predicati locativi. Viene messo in evidenza anche il fatto che in serbo si verifica un maggiore uso di verbi di postura in quanto questi verbi, oltre ad indicare la posizione del corpo, possono esprimere anche la localizzazione statica di entità animate e inanimate. Al contrario, in italiano il loro uso è più limitato e per esprimere situazioni statiche, in particolare in presenza di soggetti inanimati, si ricorre a verbi neutri come essere, stare, rimanere, trovarsi e altri. La ricerca, basata su un corpus di testi letterari paralleli, si prefigge lo scopo di fare luce sulle differenze e le somiglianze tra l'italiano e il serbo riguardo all'uso di questo particolare gruppo di verbi, nonché di segnalare i diversi modi in cui possono essere tradotti da una lingua all'altra.
\end{abstract}

Parole chiave: italiano, serbo, localizzazione statica, predicati locativi, verbi neutri.

\begin{abstract}
This paper analyzes, from a contrastive perspective, the following salient verbs and related verbal expressions indicating bodily position in Italian and Serbian: sedere, stare seduto vs sedeti, stare in piedi vs stajati and giacere, stare sdraiato/disteso vs ležati. We examine the semantic properties of these verbs in the two languages, with particular regard to their use as locative predicates. We also point out the fact that posture verbs are used more broadly in Serbian, since, beside indicating the position of the body, they can also express the static location of animate and inanimate entities. In Italian, however, their use is more limited and, in order to express static situations, in particular in the presence of inanimate subjects, Italian relies on neutral verbs such as essere, stare, rimanere, trovarsi etc. The research, based on a parallel corpus of literary texts, aims to shed light on the differences and similarities between Italian and Serbian regarding the use of this particular group of verbs, as well as to point out the various ways in which they can be translated from one language to the other.
\end{abstract}

Keywords: Italian, Serbian, static location, locative predicates, neutral verbs.

${ }^{1}$ Il contributo è stato presentato al convegno "L'italianistica nel terzo millennio: le nuove sfide nelle ricerche linguistiche, letterarie e culturali - 60 anni di studi italiani all'Università "SS. Cirillo e Metodio" di Skopje (Skopje 27-28 settembre 2019). 


\section{Introduzione}

Da alcuni decenni i verbi di postura (in seguito: VP) costituiscono un interessante e importante oggetto di studio, soprattutto nell'ambito di discipline linguistiche quali la linguistica tipologica e la linguistica contrastiva. I vari studi dedicati ai VP in lingue sia vicine che distanti dal punto di vista tipologico (Ameka \& Levinson, ${ }^{2}$ Newman ${ }^{3}$ e i lavori ivi presentati) dimostrano che le lingue differiscono notevolmente per il modo in cui i parlanti usano i VP per localizzare un'entità nello spazio, ovvero per esprimere significati locativi. I VP sono anche stati oggetto di vari studi contrastivi: svedese-italiano (Svensson), ${ }^{4}$ francese-serbo (Stosic \& Sarda), ${ }^{5}$ svedese-inglese, tedesco, francese e finlandese (Viberg), ${ }^{6}$ e inoltre, sono stati ampiamente studiati e descritti in varie lingue, soprattutto quelle germaniche, quali l'olandese (Lemmens) ${ }^{7}$ e l'inglese (Newman \& Rice, ${ }^{8}$ Newman). ${ }^{9}$

Benché l'italiano e il serbo differiscano notevolmente riguardo al modo in cui usano i VP, questi verbi sono stati finora un argomento completamente trascurato negli studi contrastivi delle due lingue. Prendendo spunto dal lavoro di Stosic e Sarda ${ }^{10}$ dedicato ai diversi modi di esprimere la localizzazione statica in francese e in serbo, tra i quali figura anche l'uso dei VP in funzione di predicati locativi, in questo studio intendiamo analizzare da un punto di vista contrastivo i VP in italiano, un'altra lingua romanza, e in serbo. La nostra ricerca, basata su un corpus parallelo di testi letterari, ha lo scopo di fare luce sulle differenze e le somiglianze esistenti tra l'italiano e il serbo riguardo all'uso di questo particolare gruppo di verbi, nonché di illustrare i loro possibili equivalenti traduttivi nelle due lingue.

${ }^{2}$ Felix K. Ameka \& Stephen C. Levinson, "The typology and semantics of locative predicates: posturals, positionals, and other beasts", Linguistics, 45, 2007, pp. 847-871.

${ }^{3}$ John Newman (ed.), The Linguistics of Sitting, Standing, and Lying, John Benjamins, Amsterdam/Philadelphia, 2002a; John Newman, “A cross-linguistic overview of the posture verb 'sit', 'stand' and 'lie'", in The linguistics of sitting, standing and lying, ed. J. Newman, John Benjamins, Amsterdam/Philadelphia, 2002b, pp. 1-24.

${ }^{4}$ Per la presente ricerca abbiamo avuto a disposizione solo l'abstract del suddetto lavoro, reperibile all'indirizzo http://hdl.handle.net/2077/16632. Data di ultima consultazione: 25/2/2020.

${ }^{5}$ Dejan Stosic \& Laura Sarda, "The Many Ways to be Located in French and Serbian: The Role of Fictive Motion in the Expression of Static location", in Space and Time in Language and Literature, eds. M. Brala Vukanovic \& L. Gruic Grmusa, Cambridge Scholar Publishing, Newcastle, 2009, pp. 39-60.

${ }^{6}$ Ake Viberg, "Posture Verbs. A Multilingual Contrastive Study", Languages in contrast 13 (2), 2013, pp. 139-169.

${ }^{7}$ Maarten Lemmens, "The Semantic Network of Dutch Posture Verbs", The Linguistics of Sitting, Standing, and Lying, ed. J. Newman, John Benjamins, Amsterdam/Philadelphia, 2002, pp. 103-139.

${ }^{8}$ John Newman \& Sally Rice, "Patterns of usage for English SIT, STAND, AND LIE: A cognitively-inspired exploration in corpus linguistics", Cognitive Linguistics, 15 (3), 2004, pp. 351-396.

${ }^{9}$ Newman, John, "English Posture Verbs. An Experientally Grounded Approach", Annual Review of Cognitive Linguistics, 2009, pp. 30-57.

${ }^{10}$ Dejan Stosic \& Laura Sarda, op. cit, pp. 39-60. 


\section{Verbi di postura}

Come segnalato da Stosic e Sarda, ${ }^{11}$ quasi tutte le lingue possiedono un gruppo di verbi che esprimono le tre principali posizioni del corpo. L'italiano ha i seguenti verbi e perifrasi verbali: sedere, stare seduto; giacere, stare sdraiato/ disteso/coricato e l'espressione stare in piedi, ai quali in serbo corrispondono, rispettivamente, i verbi: sedeti, ležati, stajati.

Osservando le forme di questi verbi e perifrasi verbali, possiamo notare che in serbo tutti e tre i VP presentano solo una forma semplice (monorematica). In italiano, al contrario, non esiste un equivalente diretto del verbo stajati, ovvero manca un verbo semplice corrispondente. Se è necessario specificare la posizione eretta di un soggetto, questa viene espressa in posizione esterna rispetto al verbo con l'aggiunta dell'elemento lessicale in piedi. Nella lingua di tutti i giorni anche agli altri due verbi, sedere e giacere (che appartiene al registro letterario ed ha un uso ristretto), si preferiscono le perifrasi formate dai verbi stare ed essere più il participio passato.

Come osserva Ross, "nelle lingue romanze mancano i verbi semplici per indicare la postura" 12 e, citando Newman, aggiunge che "tale mancanza è da collegare alla forte staticità dei verbi di postura, che proprio per questo motivo sono considerati meno tipici per la classe verbale, e il ricorso ad aggettivi o participi passati è consistente con il carattere periferico di questo tipo di verbo". ${ }^{13}$ Nella seguente tabella sono riportate le forme dei VP in inglese, serbo, italiano, francese e spagnolo.

Tabella 1. Verbi di postura in inglese, serbo, italiano, francese e spagnolo.

\begin{tabular}{|c|c|c|c|c|}
\hline inglese & serbo & italiano & francese & spagnolo \\
\hline sit & sedeti & $\begin{array}{c}\text { stare seduto } \\
\text { sedere }\end{array}$ & être assis & estar sentado \\
\hline lie & ležati & $\begin{array}{c}\text { stare sdraiato } \\
\text { (giacere) }\end{array}$ & $\begin{array}{c}\text { être couché } \\
\text { (gésir) }\end{array}$ & $\begin{array}{c}\text { estar echado } \\
\text { (yacer) }\end{array}$ \\
\hline stand & stajati & stare in piedi & être debout & estar de pie \\
\hline
\end{tabular}

${ }^{11}$ Ivi, p. 44.

${ }^{12}$ Dolores Ross, "Verbi in serie: una prospettiva tipologica", in Studi in ricordo di Carmen Sànchez Montero, vol. 2, eds. G. Benelli \& G. Tonini, EUT Edizioni Università di Trieste, Trieste, 2006, p. 456.

13 "The lack of a simple verbal category is relatable to the strong stativity of the three posture verbs (...). As such, they are less verb-like and being encoded as adjectives or past-participles is consistent with this." - John Newman, "A cross-linguistic overview of the posture verb 'sit', 'stand' and 'lie",", in The linguistics of sitting, standing and lying, ed. J. Newman, John Benjamins, Amsterdam/Philadelphia, 2002b, p. 4. 
Sia in italiano che in serbo la funzione prototipica dei VP è di descrivere la posizione del corpo di un soggetto animato: ${ }^{14}$

(1a) Stava seduta su una panchina del cortile.

(1b) Sedela je na jednoj klupi u dvorištu.

(2a) Il ragazzino sta sdraiato sul divano.

(2b) Dečak leži na kauču.

(3a) Tutti i posti erano occupati, dovevamo stare in piedi.

(3b) Sva mesta su bila zauzeta, morali smo da stojimo.

In italiano l'uso dei VP è più limitato rispetto al serbo in quanto possono apparire solo con soggetti animati (fa eccezione il verbo giacere; p.e. - le pratiche dell'affare giacciono negli uffici; però, il suo uso con soggetti inanimati non è frequente). Nel caso di soggetti inanimati l'italiano ricorre ai verbi neutri (essere, stare, restare) $\mathrm{e}$, come si vedrà più avanti, preferisce usare i verbi neutri anche con quelli animati, a meno che non sia necessario specificare la posizione del corpo.

In serbo, invece, i VP hanno un ampio uso e vengono impiegati sia con soggetti animati che con quelli inanimati. Fa eccezione il verbo sedeti 'sedere', il cui uso è limitato solo ai soggetti animati.

Gli esempi da (4) a (6) illustrano l'uso dei VP serbi con un soggetto inanimato:

(4a) Knjige stoje na polici.

Libri stanno in piedi PREP scaffale.

(4b) I libri sono sullo scaffale.

(5a) Kraj puta leži gomila đubreta.

PREP strada sta sdraiato mucchio rifiuti-SOST. GEN.

(5b) Sul bordo della strada c'è un mucchio di rifiuti.

(6a) *Jakna sedi na stolici. Giacca sta seduta PREP sedia.

(6b) *La giacca sta seduta sulla sedia.

Gli esempi sopraccitati dimostrano, tra l'altro, che l'italiano e il serbo differiscono per il modo in cui viene concettualizzata ed espressa una situazione statica. I parlanti serbofoni concettualizzano anche i soggetti inanimati in termini di postura umana.

${ }^{14}$ Gli esempi da (1) a (9) sono stati compilati dalla stessa autrice e poi verificati con altri parlanti nativi. 
In serbo il significato posizionale, prototipico dei VP, è spesso intrecciato con quello spaziale (locativo) e, come illustrano gli esempi da (7) a (9), i VP vengono impiegati per esprimere sia la posizione che la localizzazione di un'entità, assumendo spesso la funzione di verbo locativo, prevalentemente del verbo biti 'essere', ma anche nalaziti se 'trovarsi':

(7a) Sedim u kući i gledam tv.

Sto seduto PREP casa e guardo tv.

(7b) $\underline{\text { Sto }}$ a casa e guardo la tv.

(8a) U krevecu leži malo dete. PREP lettino è sdraiato piccolo bambino.

(8b) Nel lettino c'è un bambino piccolo.

(9a) Neki čovek stoji ispred kapije. Un uomo sta in piedi PREP portone.

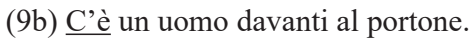

A differenza degli esempi da (1) a (3), in cui prevale il significato posizionale dei VP e la postura è specificata sia in italiano che in serbo, negli esempi da (7) a (9), in cui i VP serbi possiedono anche un significato locativo, l'italiano esprime solo il concetto di presenza/esistenza di un soggetto, senza specificarne la postura. Infatti, come si vede negli esempi, i VP serbi sono resi in italiano con il verbo neutro stare e la costruzione esistenziale $c$ 'è / ci sono.

Come osservato anche da Stosic e Sarda, ${ }^{15}$ i VP serbi hanno assunto molti significati metaforici, astratti e idiomatici (p.e. Svako veče sedim sa svojim prijateljima 'Passo tutte le serate con i miei amici'; lett. 'Sto seduto tutte le serate con i miei amici'), i quali non verranno trattati in questo lavoro.

\section{Analisi del corpus}

Per poter documentare l'uso dei VP nelle due lingue, abbiamo svolto un'analisi quantitativa e qualitativa dei VP su un corpus costituito da cinque romanzi di autori contemporanei italiani e le loro rispettive traduzioni in serbo e da cinque romanzi di autori contemporanei serbi e le loro traduzioni in italiano. Abbiamo tratto questi romanzi da un corpus di testi letterari paralleli, descritto in Moderc. ${ }^{16}$ Le occorrenze dei VP riscontrate nei romanzi originali e nelle loro traduzioni sono mostrate nella tabella 2 .

\footnotetext{
${ }^{15}$ Dejan Stosic \& Laura Sarda, op. cit., p. 46.

${ }^{16}$ Saša Moderc, "Su un modo di tradurre l'avverbio serbo "inače" in italiano: il caso dell'equivalente "altrimenti"”, Italica Belgradensia, 1, 2015, pp. 61-79.
} 
Tabella 2. Occorrenze dei VP distribuite su soggetti animati e inanimati nelle versioni originali e nelle traduzioni dei romanzi presi in analisi.

\begin{tabular}{|c|c|c|c|c|}
\hline $\begin{array}{c}\text { Verbo di } \\
\text { postura }\end{array}$ & Orig. serbo & Trad. italiana & Orig. italiano & Trad. serba \\
\hline Sogg. animato & 229 & 141 & 115 & 110 \\
\hline Sogg. inanimato & 67 & 3 & $\varnothing$ & $\varnothing$ \\
\hline Totale & 296 & 144 & 115 & 110 \\
\hline
\end{tabular}

I dati riportati nella tabella 2 dimostrano chiaramente una prevalenza di occorrenze dei VP nei romanzi serbi, pari a più del doppio delle occorrenze dei VP nei romanzi italiani. Infatti, per i 296 casi di VP in serbo abbiamo 115 casi di VP in italiano.

Inoltre, le traduzioni dal serbo all'italiano (terza colonna) mostrano che solo la metà, ovvero un po' meno della metà dei VP serbi sono stati tradotti in italiano con un VP, e in quasi tutti i casi i VP appaiono con un soggetto animato. Solo in tre casi è stato usato il verbo giacere con un soggetto inanimato.

Al contrario, le traduzioni dall'italiano al serbo (quinta colonna) mostrano che i VP italiani sono stati tradotti in serbo con un VP in quasi tutti i casi.

Nella seguente tabella sono riportati i diversi tipi di verbo usati nelle traduzioni dal serbo all'italiano.

Tabella 3. Traduzione dei VP serbi in italiano.

\begin{tabular}{|c|c|c|c|}
\hline $\begin{array}{c}\text { Tipo di verbo nelle } \\
\text { traduzioni italiane }\end{array}$ & Soggetto animato & Soggetto inanimato & Totale \\
\hline Verbo di postura & $141(48 \%)$ & $3(1 \%)$ & $144(49 \%)$ \\
\hline Verbo neutro & $61(20,5 \%)$ & $31(10,5 \%)$ & $92(31 \%)$ \\
\hline Altro verbo & $21(7 \%)$ & $27(9 \%)$ & $48(16 \%)$ \\
\hline Omissione & $9(3 \%)$ & $3(1 \%)$ & $12(4 \%)$ \\
\hline
\end{tabular}

Come si può osservare nella tabella 3 , i VP serbi usati con un soggetto animato sono stati tradotti in italiano con un VP nel 48\% dei casi, nel 20,5\% dei casi con un verbo neutro, nel $7 \%$ con un altro tipo di verbo, e solo in pochi casi sono stati omessi nella traduzione. Il verbo più tradotto con un VP è il verbo sedeti 'sedere', mentre il verbo meno tradotto con un VP è stajati 'stare in piedi'.

Gli esempi da (10) a (18) illustrano i diversi modi in cui i VP serbi usati con soggetti animati sono stati tradotti in italiano. Negli esempi (10) e (11) i VP serbi sono stati tradotti in italiano con un VP, negli esempi (12)-(15) con un verbo neutro o la struttura esistenziale $c$ 'è / ci sono, nell'esempio (16) è stato usato un altro tipo di verbo e negli esempi (17) e (18) i VP serbi sono stati omessi nella traduzione. 
(10a) Tek tada je pošao dalje pogledom i video da je ta knjiga na krilu čoveka koji samo napola leži a napola sedi, naslonjen na svoj kovčežić. (Andrić)

(10b) Solo allora fra Petar spinse oltre lo sguardo e vide che il libro era in grembo a un uomo che stava per metà sdraiato e per metà seduto, appoggiato alla sua valigetta.

(11a) Najčešće mi se čini da ne stojim već da klizim, ... (Albahari)

(11b) Spesso mi pare di non stare in piedi ma di scivolare...

(12a) Počeo je da izbegava prijeme, balove i odlaske u operu, jer tamo je neuljudno sedeti sa cilindrom golubije boje na glavi... (Gatalica)

(12b) Cominciò ad evitare i ricevimenti, i balli e le serate all'opera, perché in quei luoghi non era opportuno stare con il cilindro color colombo in testa...

(13a) Ležala je na ostacima nedojedene ribe kao pustinjski pas na kostima ulova, kada je Masudi prišao. (Pavić)

(13b) Se ne stava sopra gli avanzi del pesce come un cane del deserto sulle ossa della preda quando Masudi si avvicinò.

(14a) Dugo je još potom stajala nepomična, ... (Gatalica)

(14b) E poi era rimasta a lungo immobile, ...

(15a) Pred okruglim prozorom jedne od kabina prve klase stajao je putnik. (Gatalica)

(15b) Davanti all'oblò di una delle cabine di prima classe c'era un passeggero.

(16a) Kad se okrenuo da pođe, pred njim je stajao Haim. (Andrić)

(16b) Quando si voltò per andarsene, si vide davanti Haim.

(17a) Dubrovčani su ga upamtili kako stoji uvek na istom mestu, ... (Pavić)

(17b) Gli abitanti di Dubrovnik lo ricordano sempre nello stesso posto, ...

(18a) Ali on je sedeo, čitao dokumenta ... (Gatalica)

(18b) Ma lui stava leggendo dei documenti ...

Questi dati confermano la nostra osservazione che l'italiano tende a privilegiare i verbi neutri per esprimere la posizione del corpo anche in riferimento a soggetti animati. Infatti, come si può notare nella tabella 3 , nel 20,5\% dei casi al posto di un VP nella traduzione italiana sono stati usati verbi neutri (essere, stare, rimanere, trovarsi) o la costruzione esistenziale c'è / ci sono.

I VP serbi usati con soggetti inanimati sono stati tradotti con un verbo neutro nel 10,5\% dei casi (in 3 occorrenze con il VP giacere), nel 9\% dei casi con un altro verbo, spesso un verbo di movimento fittizio, come in (23), con un elemento 
lessicale (un aggettivo, un participio), come in (24) e (25), o sono stati omessi nella traduzione, come in (26). I seguenti esempi illustrano i diversi modi in cui i VP serbi usati con soggetti inanimati sono stati tradotti in italiano:

(19a) Sedeli smo za stolom u trpezariji, preda mnom je ležao papir (...), pred njom je, (...), stajao mikrofon, ... (Albahari)

(19b) Eravamo seduti al tavolo della sala da pranzo, davanti a me stava un foglio (...), davanti a lei, (...), stava il microfono, ...

(20a) Tamo je stajala jabuka i on je čekao da vetar dune. (Pavić)

(20b) Là c'era un albero di mele e lui attese che tirasse il vento.

(21a) (...) a sultan Bajazit preneo je telo nesrećnog brata odmetnika i sahranio ga u Brusi, gde i danas stoji njegovo turbe. (Andrić)

(21b) (...) e Baiazet aveva ottenuto il corpo dell'infelice fratello ribelle e lo aveva fatto seppellire a Brussa, dove ancor oggi si trova il suo mausoleo.

(22a) Čitav objekat je jedna spirala koja svojim kosim ravnima leži na zemlji ukopana. (Basara)

(22b) L'intero manufatto è una spirale che con i suoi piani inclinati poggia sul terreno interrato.

(23a) Ali to more nije ležalo u vidiku: ono je stajalo u njemu uspravno kao zavesa ... (Pavić)

(23b) Tuttavia questo mare non si stendeva lungo la linea dell'orizzonte, ma si ergeva in verticale come una tenda ...

(24a) U tornju, nad kartama Atlantskog okeana, stajali su polusavijeni okamenjeni oficiri... (Gatalica)

(24b) Sulla torretta di pilotaggio, fissi sopra le carte nautiche dell'oceano Atlantico, c'erano alcuni ufficiali letteralmente irrigiditi, ...

(25a) U obućarskoj radnji je stajalo mnogo modela visokog sjaja. (Gatalica)

(25b) Nel negozio erano esposti molti modelli di alto splendore.

(26a) (...) sada, ovde, nisam siguran da sam za to vreme napisao bilo šta na listu papira koji je ležao preda mnom. (Albahari)

(26b) (...) ora, qui, non sono sicuro di aver scritto, in quel lasso di tempo, qualcosa su quel foglio di carta davanti a me.

In base all'analisi del corpus, possiamo notare che l'italiano ricorre a diverse strategie per evitare di usare un VP, non solo con soggetti inanimati, con i quali in generale non si usano i VP, ma anche con quelli animati, come abbiamo 
visto nel 30,5\% dei casi. Va detto anche che i risultati della nostra analisi non differiscono molto dai risultati a cui sono arrivati Stosic e Sarda ${ }^{17}$ paragonando i $\mathrm{VP}$ in francese e in serbo. Infatti, essendo entrambi lingue romanze, il francese e l'italiano si comportano in maniera simile riguardo all'uso dei VP.

La nostra analisi rivela anche altri dati interessanti. Abbiamo già visto in precedenza che nei cinque romanzi italiani sono presenti 115 occorrenze di VP, tutti usati con soggetti animati e quasi tutti tradotti in serbo con un VP. Però, è interessante e significativo il fatto che nelle traduzioni in serbo appaiano altri 174 VP che non figurano nei romanzi italiani. In altre parole, sono state registrate 115 occorrenze di VP nei romanzi italiani e 289 occorrenze nelle traduzioni in serbo, dato che molti verbi neutri italiani sono stati tradotti in serbo con un VP. Quindi, il numero dei VP usati nelle traduzioni in serbo supera di gran lunga il numero dei VP presenti nelle versioni originali in italiano. Questo dato illustra chiaramente la differenza che esiste tra le due lingue riguardo alla frequenza d'uso dei VP e alla loro importanza nell'espressione di situazioni statiche nelle due lingue.

Gli esempi da (27) a (33) illustrano diversi tipi di verbi italiani (per lo più verbi neutri) tradotti in serbo con un VP:

(27a) Ora non cercava più: stava lì, rimandando di giorno in giorno il suo ritorno in Italia,... (Duranti)

(27b) Sada više nije tražio; sedeo je tu, odlažući svoj povratak u Italiju iz dana u dan, ...

(28a) Eravamo a letto. (Ammaniti 2001)

(28b) Ležali smo u krevetu.

(29a) Padre Pluche se ne stava col naso appiccicato ai vetri, incantato. (Baricco)

(29b) Otac Pluš je stajao nosa priljubljenog uza staklo, opčinjen.

(30a) Simon Berish rimase immobile per un lungo momento, osservando la scena. (Carrisi)

(30b) Beriš je prilično dugo nepomično sedeo i posmatrao scenu.

(31a) Un vero letto era piazzato al centro, ... (Carrisi)

(31b) U sredini sobe stajao je pravi krevet ...

(32a) In fondo, davanti a una chiesa moderna, c'era un grosso Suv Mercedes. (Ammaniti 2010)

(32b) Na kraju ulice, ispred novoizgrađene crkve, stajao je veliki mercedes.

\footnotetext{
${ }^{17}$ Dejan Stosic \& Laura Sarda, op. cit., pp. 39-60.
} 
(33a) Quella di Mario era l'alloggio del custode e sorgeva sul ripiano più basso del giardino... (Duranti)

(33b) Mariova kuća bila je zapravo nastojnički stan i stajala je na najnižoj ravni bašte...

\section{Conclusioni}

L'analisi effettuata in questa ricerca rivela tanto le somiglianze quanto le differenze tra l'italiano e il serbo riguardo al modo in cui queste due lingue usano i VP. Come si è visto, le somiglianze tra le due lingue si notano soprattutto nell'uso prototipico dei VP, mentre le differenze riguardano l'uso di questi verbi in funzione di predicati locativi.

Il serbo usa con maggiore frequenza i VP in quanto questi verbi, oltre ad indicare la posizione del corpo, possono indicare anche la localizzazione statica di un'entità animata o inanimata. Dall'altra parte, l'italiano è caratterizzato da un uso più limitato dei VP, dato che non possono comparire con entità inanimate. Per esprimere una situazione statica, in particolare in presenza di entità inanimate, ma spesso anche in presenza di quelle animate, l'italiano ricorre a verbi neutri come essere, stare, rimanere, trovarsi e altri.

In generale, i risultati della nostra analisi conducono alla conclusione che nel dominio della localizzazione statica il serbo presta più attenzione alla posizione assunta da un'entità, e usa i VP, ${ }^{18}$ mentre l'italiano si limita a segnalare la localizzazione o la presenza/esistenza di un'entità usando i verbi neutri o la costruzione esistenziale $c$ 'è / ci sono. L'italiano ricorre ai VP per lo più quando è necessario specificare la postura o quando la postura che un'entità assume non risulta facilmente inferibile dal contesto.

Con il presente lavoro si è voluto dare un contributo alle ricerche contrastive tra l'italiano e il serbo, sperando che possa rappresentare un punto di partenza e di riferimento per successive ricerche incentrate sui modi di esprimere le relazioni spaziali statiche o dinamiche nelle due lingue.

\section{BIBLIOGRAFIA}

Ameka Felix K. \& Levinson Stephen C. "The typology and semantics of locative predicates: posturals, positionals, and other beasts", Linguistics, 45, 2007, pp. 847-871.

Lemmens Maarten, "The Semantic Network of Dutch Posture Verbs", The Linguistics of Sitting, Standing, and Lying, ed. J. Newman, John Benjamins, Amsterdam/Philadelphia, 2002, pp. 103-139.

Moderc Saša, "Su un modo di tradurre l'avverbio serbo "inače” in italiano: il caso dell'equivalente "altrimenti"”, Italica Belgradensia, 1, 2015, pp. 61-79.

\footnotetext{
${ }^{18}$ Cfr. Dejan Stosic \& Laura Sarda, op. cit., p. 50.
} 
Newman John (ed.), The Linguistics of Sitting, Standing, and Lying, John Benjamins, Amsterdam/Philadelphia, 2002a.

Newman John, "A cross-linguistic overview of the posture verb 'sit', 'stand' and 'lie"", in The linguistics of sitting, standing and lying, ed. J. Newman, John Benjamins, Amsterdam/Philadelphia, 2002b, pp. 1-24.

Newman John \& Rice Sally, "Patterns of usage for English SIT, STAND, AND LIE: A cognitively-inspired exploration in corpus linguistics", Cognitive Linguistics, 15 (3), 2004, pp. 351-396.

Newman, John, "English Posture Verbs. An Experientally Grounded Approach", Annual Review of Cognitive Linguistics, 2009, pp. 30-57.

Ross Dolores, "Verbi in serie: una prospettiva tipologica", in Studi in ricordo di Carmen Sànchez Montero, vol. 2, eds. G. Benelli \& G. Tonini, EUT Edizioni Università di Trieste, Trieste, 2006, pp. 453-464.

Stosic Dejan \& Sarda Laura, "The Many Ways to be Located in French and Serbian: The Role of Fictive Motion in the Expression of Static location", in Space and Time in language and Literature, eds. M. Brala Vukanovic \& L. Gruic Grmusa, Cambridge Scholars Publishing, Newcastle, 2009, pp. 39-60.

Svensson Kristina, Uno studio contrastivo svedese-italiano sui verbi svedesi stå, sitta e ligga, Doctoral thesis, Gothenburg, University of Gothenburg, 2005. www.http://handle.net/2077/16632.

Viberg Ake, "Posture Verbs. A Multilingual Contrastive Study", Languages in contrast 13 (2), 2013, pp. 139-169.

\section{FONTI}

Albahari, David, Mamac, Beograd, Narodna knjiga, 1996.

Albahari, David, L'esca, trad. A. Parmeggiani, Rovereto, Zandonai, 2008.

Ammaniti Niccolò, Io non ho paura, Torino, Einaudi, 2001.

Amaniti Nikolo, Ne bojim se, trad. D. Todorović-Lakava, Beograd, Plato, 2002.

Ammaniti, Niccolò, Io e te, Torino, Einaudi, 2010.

Amaniti Nikolo, Ja i Ti, trad. M. Radosavljević e A. Levi, Beograd, Plato, 2011.

Andrić, Ivo, Prokleta avlija, Novi Sad, Matica srpska, 1986.

Andric, Ivo, La Corte del diavolo, trad. L. Costantini, Milano, Adelphi, 1992.

Baricco Alessandro, Oceano mare, Milano, Rizzoli, 1993.

Bariko, Alesandro, Okean more, trad. E. Vasiljević, Beograd, Filip Višnjić, 1997.

Basara Svetislav, Fama o biciklistima, Beograd, Dereta, 1987.

Basara, Svetislav, Quel che si dice dei Ciclisti Rosacroce, trad. M. R. Leto, Milano, Anfora, 2005. 
Carrisi, Donato, L'ipotesi del male, Milano, Longanesi, 2013.

Karizi, Donato, Vladar iz senke, trad. B. Kukoleča, Beograd, Vulkan, 2013.

Duranti, Francesca, La casa sul lago della luna, Milano, Rizzoli, 1984.

Duranti, Frančeska, Kuća na mesečevom jezeru, trad. I. Klajn, Beograd, Nolit, 1991.

Gatalica, Aleksandar, Vek. Sto jedna povest jednog veka, Beograd, Stubovi kulture, 1999.

Gatalica, Aleksandar, Secolo. Cento e una storia di un secolo, trad. S. Ferrari e A. Džankić, Reggio, Emilia, Edizioni Diabasis, 2008.

Pavić, Milorad, Hazarski rečnik, Beograd, Prosveta, 1984.

Pavić, Milorad, Dizionario dei Chazari, trad. B. Ničija, Milano, Garzanti, 1988.

Наташа Јанићијевић

\section{ГЛАГОЛИ ПОЛОЖАЈА У ИТАЛИЈАНСКОМ И СРПСКОМ ЈЕЗИКУ \\ (Резиме)}

У раду се описују, анализирају и пореде глаголи положаја у италијанском и српском језику. Резултати анализе спроведене на паралелном корпусу књижевних текстова показују да је употреба глагола положаја чешћа у српском језику, будући да, поред тога што означавају положај тела, они могу да изразе и статичку локализацију како живог, тако и неживог субјекта. У италијанском језику употреба ових глагола је ограниченија јер се не могу јавити уз неживе субјекте, те се, за означавање статичких ситуација у присуству неживог субјекта, а често и живог, прибегава неутралним глаголима. Уопште узевши, код изражавања статичке локализације српски језик поклања већу пажњу начину на који је субјекат позициониран и употребљава глаголе положаја, док италијански ставља нагласак на локализацију или на само присуство субјекта користећи неутралне глаголе или егзистенцијалну структуру c'è / ci sono 'има', 'постоји', 'налази се'. Глаголи положаја се користе углавном када је потребно спецификовати положај који заузима субјекат или када се начин на који је субјекат позициониран не може лако разазнати на основу ширег контекста.

Кључне речи: италијански, српски, статичка локализација, предикати с локативним значењем, неутрални глаголи.

Примљено 11. маја 2020, прихваћено за објављивање 10. децембра 2020. године. 